\title{
A new genus of Neotropical Chrysopini (Neuroptera: Chrysopidae)
}

\author{
FRANCISCO SOSA ${ }^{1} \&$ SERGIO DE FREITAS ${ }^{2}$ \\ ${ }^{1}$ Universidad Centroccidental "Lisandro Alvarado", Museo Entomológico “Dr. José Manuel Osorio” (UCOB), Barquisimeto, Lara, . \\ E-mail:fransodu73@hotmail.com \\ ${ }^{2}$ Universidade Estadual Paulista, Jaboticabal, São Paulo, Brazil (deceased)
}

\begin{abstract}
Titanochrysa Sosa \& Freitas is a new genus of Neotropical Chrysopini (Chrysopidae: Chrysopinae) recorded from Costa Rica, Venezuela and Brazil. Titanochrysa gen. nov. shares several external and genitalic characters with Ceraeochrysa Adams, 1982; Chrysopodes Navás, 1913; Cryptochrysa Freitas \& Penny, 2000; Parachrysopiella Brooks \& Barnard, 1990 and Ungla Navás 1914. It may be distinguished from those genera by its very long sternite $8+9$, sternites $2-8$ usually with microtholi, male genitalia with the dorsal surface of the arcessus striated, gonosaccus well-developed, bearing elongate gonosetae and microsetae, and a spoon-like gonapsis. Herein, Titanochrysa circumfusa (Burmeister, 1939) [= Chrysopodes circumfusa (Burmeister)] comb. nov. and Titanochrysa pseudovaricosa (Penny) [= Ceraeochrysa pseudovaricosa Penny, 1998] comb. nov. were identified; Titanochrysa ferreirai Sosa \& Freitas sp. nov. and Titanochrysa trespuntensis Sosa \& Freitas sp. nov. were described. The external morphology, and male and female genitalia of all these species are described. Titanochrysa circumfusa (Burmeister, 1939) comb. nov. is recorded for the first time from Venezuela.
\end{abstract}

Key words: Chrysopinae, Chrysopini, green lacewing, predator

\section{Resumen}

Titanochrysa Sosa \& Freitas es un nuevo género Neotropical de Chrysopini (Chrysopidae: Chrysopinae) con registros para Costa Rica, Venezuela y Brasil. Titanochrysa gen. nov. comparte varias características externas y de la genitalia con Ceraeochrysa Adams, 1982; Chrysopodes Navás, 1913; Cryptochrysa Freitas \& Penny, 2000; Parachrysopiella Brooks \& Barnard, 1990 y Ungla Navás, 1914. Se diferencia de aquellos géneros por presentar el esternito 8+9 muy largo, esternitos 2-8 generalmente con microtholi, genitalia del macho con superfície dorsal del arceso estriada, gonosaco desarrollado con largas gonosetas y microsetas, gonapsis dilatada basalmente con ápice serreado. Aquí: Titanochrysa circumfusa (Burmeister, 1939) [= Chrysopodes circumfusa (Burmeister)] comb. nov. y Titanochrysa pseudovaricosa (Penny, 1998) [= Ceraeochrysa pseudovaricosa Penny, 1998] comb. nov. fueron identificadas y Titanochrysa ferreirai Sosa \& Freitas sp. nov., y Titanochrysa trespuntensis Sosa \& Freitas sp. nov. descritas. La morfología externa, así como la genitalia del macho y la hembra son descritos. Titanochrysa circumfusa (Burmeister, 1939) comb. nov. es reportada para Venezuela por primera vez.

Palabras clave: Chrysopinae, Chrysopini, crisópidos, depredator

\section{Introduction}

Adams (1978) organized the higher classification of Neotropical Chrysopidae including genera within tribes and subfamilies. Later, Adams (1982a, b) described Plesiochrysa Adams and Ceraeochrysa Adams, respectively. Brooks \& Barnard (1990) identified 31 genera in Chrysopini, and Freitas \& Penny (2000) added two new genera. At present, this tribe has 33 genera: nine are present in the Neotropics.

Recently, we discovered that several species have traits that do not conform to any of the described Neotropical genera; they appear to belong in a distinct, previously undescribed genus. We herein describe this genus, Titanochrysa gen. nov. We transfer two previously described species-Chrysopodes circumfusus (Burmeister, 1853) and Ceraeochrysa pseudovaricosa Penny, 1998- to the new genus, and we describe two new Titanochrysa species. 